\title{
Erratum
}

\section{Homicidal ideation causally related to therapeutic medications}

Donald H. Marks, Peter R. Breggin and Derek Braslow

[International Journal of Risk \& Safety in Medicine 20(4) (2008), 231-240]

When this article was originally published, the following footnote was omitted from the first page:

The present paper appears simultaneously in Ethical Human Psychology and Psychiatry, Journal of the International Center for the Study of Psychiatry and Psychology and is published with permission of Springer Publishing Company, New York, NY, USA. 